\title{
A Vision based Human Robot Interface for Robotic Walkthroughs in a Biotech Laboratory.
}

\author{
Martin Wojtczyk, Giorgio Panin, \\ Claus Lenz, Thorsten Röder, Suraj Nair, \\ Erwin Roth and Alois Knoll \\ Technische Universität München \\ Department of Informatics VI \\ Robotics and Embedded Systems \\ Boltzmannstr. 3 \\ 85748 Garching b. München, Germany \\ wojtczyk@in.tum.de
}

\author{
Rüdiger Heidemann, Klaus Joeris, \\ Chun Zhang, Mark Burnett and \\ Tom Monica \\ Bayer HealthCare - Pharmaceuticals \\ Global Biological Development \\ 800 Dwight Way \\ Berkeley, CA, 94710, USA \\ rudiger.heidemann.b@bayer.com
}

\begin{abstract}
Both Robots and Personal Computers established new markets about 30 years ago and were enabling factors in $\mathrm{Au}-$ tomation and Information Technology. However, while you can see Personal Computers in almost every home nowadays, the domain of Robots in general still is mostly restricted to industrial automation. Due to the physical impact of robots, a safe design is essential, which most robots still lack of and therefore prevent their application for personal use, although a slow change can be noticed by the introduction of dedicated robots for specific tasks, which can be classified as service robots. Moreover, as more and more robots are designed as service robots, their developers face the challenge of reducing the machines' complexity and providing smart user interface methods. Ideally the robot would be able to cooperate with a human, just like another human would.
\end{abstract}

\section{Categories and Subject Descriptors}

I.4.9 [Image Processing And Computer Vision]: Applications

\section{General Terms}

Algorithms, Human Factors

\section{Keywords}

Lab Automation, Life Sciences, HRI, Model based Tracking

\section{BACKGROUND}

Our approach to service robots was driven by the idea of supporting lab personnel in a biotechnology laboratory. That resulted in the combination of a manipulator with a mobile platform, extended with the necessary sensors to carry out a complete sample management process in a mammalian cell culture plant $[1,4]$. After the initial development in Germany, the mobile manipulator was shipped to Bayer HealthCare in Berkeley, CA, USA, a global player in the sector of biopharmaceutical products, located in the San Francisco bay area. The platform was installed and successfully

Copyright is held by the author/owner(s).

HRI'09, March 11-13, 2009, La Jolla, California, USA.

ACM 978-1-60558-404-1/09/03. tested there in a pilot plant to carry out a robust and repetitive sample management process as we showed on the well known ESACT meeting [5]. Figure 1 shows the robot, as it arrived on site with laser range finders to monitor the environment and to localize itself and a manipulator with an attached gripper tool, a force/torque sensor and a camera to observe and interact with the environment while figure 2 shows the robot in the pilot plant with a bioreactor, different types of sample vials and analysis devices.

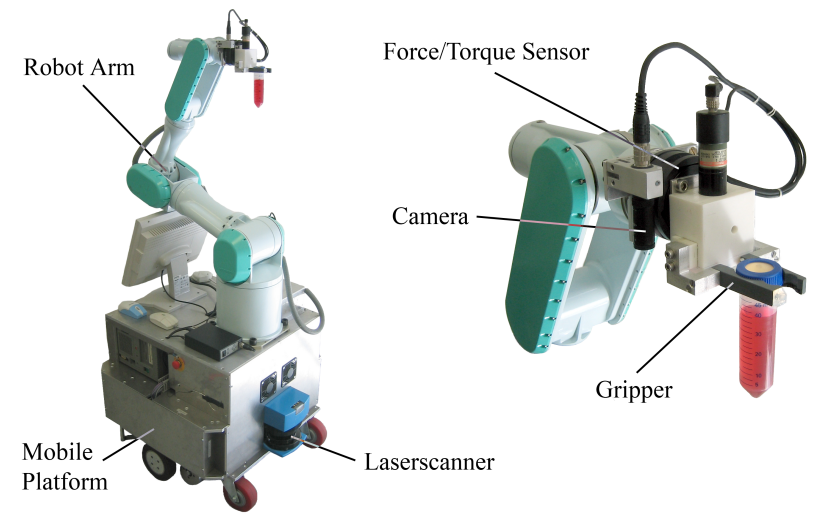

Figure 1: The mobile robot as it was shipped to the customer.

\section{CHALLENGES}

As the project progressed, two challenges were encountered. On the one hand the lab setup was not completely static. Therefore the robot should be easily adjustable to a changing environment, without the demand for a dedicated programmer. On the other hand - once installed successfully - new ideas came up for the additional use of the robot: a possibly remote user should be able to carry out (semi-)automated lab walkthroughs, for example at night, to check the status of the lab equipment such as bioreactors and pumps.

\section{SOLUTION}

In both cases - updating the robot's world model and initially teaching the lab walkthroughs or performing subse- 


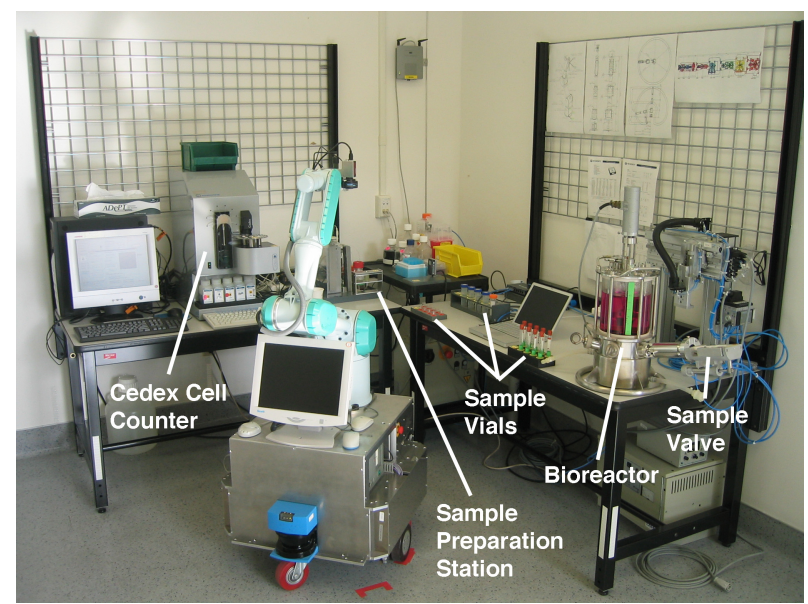

Figure 2: The robot lab for the cell counting sample management process with all necessary devices.

quent updates on them - a recent development of our computer vision group comes into play: OpenTL - an Integrated Framework for Model-based Object Tracking [3]. Figure 3 shows the generic work flow of the implemented tracking methods and 4 depicts one exemplary application of the framework for face tracking.

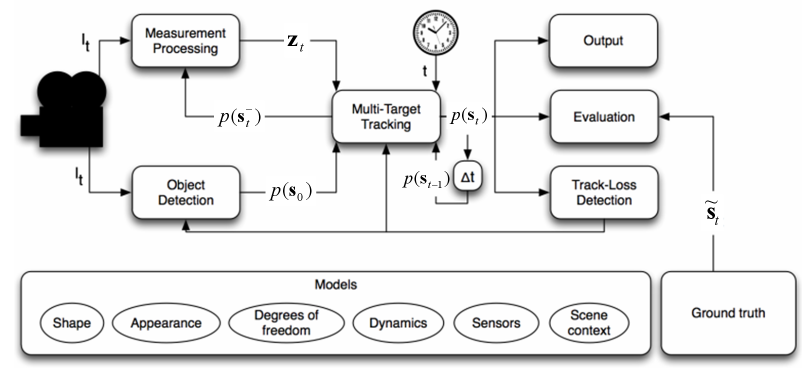

Figure 3: The flow diagram of our model-based, multi-purpose tracking library: OpenTL.

By utilizing the robots camera pointed at the teacher and by running the person and face tracker, the robot is able to follow the teacher through the lab and to pay attention to the teacher's points of interests by evaluating the pose of the head and by interpreting the teachers gestures, for example simple pointing gestures with the index finger. Constraint rules and the continuous evaluation of the laser range finders' data makes sure, that collisions with the possibly unknown environment are prohibited. The successful utilization of the person tracker has been previously shown in a real world TV Studio in Cologne, Germany [2]. This way even complex lab walkthrough sequences can be taught by regular lab personnel. Additionally, this way, lab personnel is also able to update new positions of lab devices for the implemented sample management process.

The utilization of our model based tracking library is an enourmous step towards human robot interaction in the given setup. By making the robot see and understand where a person moves and what a person is pointing at, the programming overhead for a mobile manipulator in a change-

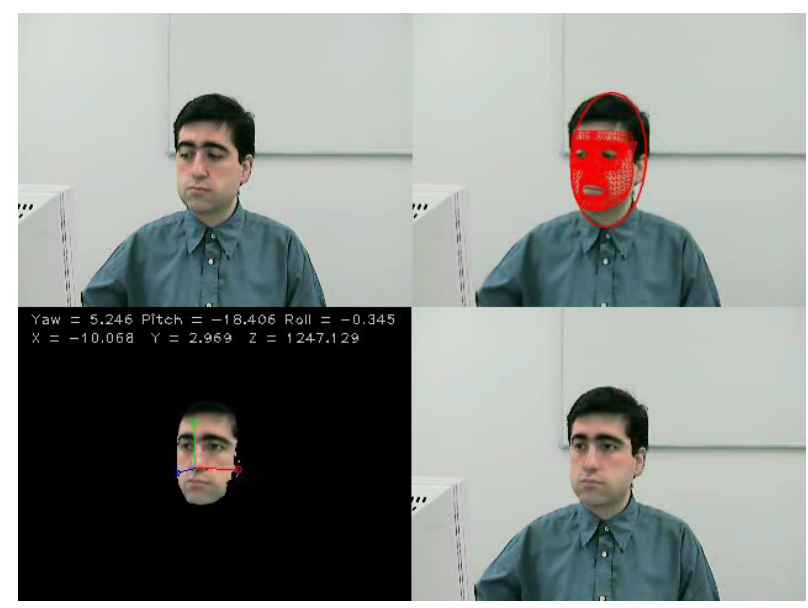

Figure 4: Template and contour based face tracking as one example of the tracking library.

able environment can be reduced dramatically, hence the robot can be used by regular lab personnel which is an important requirement to make service robots successful.

\section{REFERENCES}

[1] D. Lütkemeyer, I. Poggendorf, T. Scherer, J. Zhang, A. Knoll, and J. Lehmann. First Steps in Robot Automation of Sampling and Sample Management during Cultivation of Mammalian Cells in Pilot Scale. Biotechnology Progress, 16(5):822-828, 2000.

[2] S. Nair, G. Panin, M. Wojtczyk, C. Lenz,

T. Friedlhuber, and A. Knoll. A Multi-Camera Person Tracking System for Robotic Applications in Virtual Reality TV Studio. In 2008 IEEE/RSJ International Conference on Intelligent Robots and Systems,

Acropolis Convention Center, Nice, France, Sept. 2008. Accepted for publication.

[3] G. Panin, C. Lenz, M. Wojtczyk, S. Nair, E. Roth, T. Friedlhuber, and A. Knoll. A unifying software architecture for model-based visual tracking. In Image Processing: Machine Vision Applications. Edited by Niel, Kurt S.; Fofi, David. Proceedings of the SPIE, Volume 6813, pp. 681303-681303-14 (2008)., volume 6813 of Presented at the Society of Photo-Optical Instrumentation Engineers (SPIE) Conference, Mar. 2008.

[4] T. Scherer, I. Poggendorf, A. Schneider, D. Westhoff, J. Zhang, D. Lutkemeyer, J. Lehmann, and A. Knoll. A service robot for automating the sample management in biotechnological cell cultivations. Emerging Technologies and Factory Automation, 2003. Proceedings. ETFA '03. IEEE Conference, 2:383-390 vol.2, Sept. 2003.

[5] M. Wojtczyk, R. Heidemann, K. Joeris, C. Zhang, M. Burnett, A. Knoll, and K. Konstantinov. The Use of a Mobile Robot for Complete Sample Management in a Cell Culture Pilot Plant. In R. Smith, editor, Cell Technology for Cell Products, Proceedings of the 19th ESACT Meeting, Harrogate, UK, June 5-8, 2005, pages 543-547. Springer Netherlands, 2007. 